\title{
First impressions are lasting impressions: A primacy effect in memory for repetitions
}

\author{
GREGORY J. DIGIROLAMO and DOUGLAS L. HINTZMAN \\ University of Oregon, Eugene, Oregon
}

\begin{abstract}
Two experiments demonstrated that the encoding of a repeated object is biased toward the attributes of its first presentation. In Experiment 1, subjects saw objects five times each, but either the first presentation or the fifth presentation was the mirror reverse of the standard orientation seen on the other four trials. When recognition was tested with both orientations simultaneously, subjects reported seeing only the single mirror-reverse orientation more often if it was the first presentation than when it was the fifth presentation, and seeing only the standard orientation more often if it was presentations 1-4 than when it was presentations $2-5$. A second experiment demonstrated that this primacy effect generalized to size changes. This pattern of results is consistent with the hypothesis that top-down biases affect what subjects learn: The first representation established for a stimulus is likely to influence the encoding of subsequent repetitions.
\end{abstract}

As a stimulus becomes more familiar, the amount of attention it is given may be attenuated (Sokolov, 1963). As a consequence, additional learning about the stimulus may be impaired. An apparent example of this phenomenon is "registration without learning," in which repetitions of an item register (as demonstrated by increasing frequency judgments), but the ability to discriminate the item from similar items does not increase in a manner commensurate with the number of presentations (Hintzman \& Curran, 1995; Hintzman, Curran, \& Oppy, 1992).

To illustrate, in Experiment 2 of Hintzman et al. (1992), subjects saw individual pictures from 1 to 15 times. On the following test, each picture was presented in either the study orientation or its mirror-reverse orientation. The subjects's task was to judge the presentation frequency of the item exactly as seen, giving a frequency of zero if the test item's orientation was different from that seen at study. Frequency judgments for pictures tested in the study orientation increased monotonically with presentation frequency, but incorrect acceptance of the mirrorreversed items as old remained constant at about $39 \%$ over the entire range of study frequencies ( 1 to 15 presentations). Apparently, even though subjects knew how often an item had been repeated, they learned little more about its orientation following the first presentation.

Learning curves typically have an exponential (Estes, 1950 ) or sigmoidal form (Culler \& Girden, 1951). By con-

This research was supported by the James S. McDonnell Foundation and Pew Memorial Trusts through the support of the Center for the Cognitive Neuroscience of Attention, and National Science Foundation Grant SBR-93-19265 to D. L. Hintzman. We thank Michael Posner, John Gardiner, Art Glenberg, William Johnston, and Roddy Roediger for helpful comments on earlier drafts of this paper. Correspondence should be addressed to G. J. DiGirolamo, Department of Psychology, University of Oregon, Eugene, OR 97403 (e-mail: gjd@oregon.uoregon. edu). trast, in the Hintzman et al. (1992) experiments, the first presentation produced considerable learning of left-right orientation, but learning on subsequent presentations was anomalously slow. Hintzman and Curran (1995, Experiment 2) demonstrated this result explicitly in a forcedchoice recognition test between the mirror-reversed pictures and the studied versions of the pictures, which had appeared at different frequencies in the study list. An exponential learning curve systematically underpredicted performance at lower frequencies and overpredicted performance at higher frequencies (see Figure 5, panel b, of Hintzman \& Curran, 1995). Hintzman and Curran suggested that these results could reflect a resource allocation process. A strong familiarity signal triggered by a repeated item during study could modulate the item's encoding so that the repetition is catalogued, but further information regarding its appearance (in this case, orientation) is not stored.

In the Hintzman and Curran experiments (1995; Hintzman et al., 1992), all study presentations of a stimulus were identical. In this paper, we ask what happens if the attributes of a repeated object change during study. We predict that if two similar versions of an object are studied, the first exemplar will influence the encoding of subsequent presentations so that the changed attribute will tend not to be encoded. Such top-down processes could (1) attenuate the encoding of the attributes of a repeated stimulus; (2) impose attributes of the first version of the stimulus on encodings of later presentations, even if that attribute of the stimulus has changed; or (3) both. The result, in any case, would be a primacy effect in memory for repetitions. We tested this prediction in two experiments, one in which objects changed in left-right orientation, and one in which they changed in size.

These experiments compared the effects of early or late changes in orientation (Experiment 1) or size (Experiment 2) of a repeated object on later memory of this 
attribute. Each target object was presented five times in one of three conditions: (1) no change in orientation or size (all-same condition); (2) first presentation different from Presentations 2-5 (first-different condition); and (3) fifth presentation different from Presentations 1-4 (fifth-different condition). On the test, both versions of the object were displayed concurrently, and subjects were required to indicate the version or versions they had seen in the study phase.

\section{METHOD}

\section{Subjects}

We tested a total of 54 University of Oregon undergraduates in groups of 1 to 5 subjects $(N=27$ in Experiment 1 and $N=27$ in Experiment 2). The data of 18 other subjects ( 9 from each experiment) were excluded because they did not circle a single response for every test pair, as instructed. All subjects received course credit for participation.

\section{Design and Materials}

A subset of asymmetrical line drawings of common objects from the Snodgrass and Vanderwart (1980) stimulus set was adapted to create 126 bit-mapped images. In Experiment 1, the stimulus attribute that was changed was left-right orientation. In Experiment 2, it was size. Most objects from Experiment 1 were also used in Experiment 2. However, six items that did not fit on the computer screen when enlarged were replaced with smaller items from the same stimulus pool.

Sixty objects were randomly assigned to the three experimental conditions: 15 to the first-different condition, 15 to the fifth-different condition, and 30 to the all-same condition. The remaining 60 objects were randomly assigned for use as either repeated or nonrepeated fillers. The study list consisted of 420 presentations of objects arranged in five blocks. For Blocks 2-4, five objects from each condition were assigned to each block. The 15 objects assigned to the all-same condition that were not used in Blocks 2-4 were combined with repeated fillers to construct two buffer blocks (Blocks 1 and 5), at the beginning and end of the study list. Each block consisted of five segments, and Presentations $1-5$ of the objects were assigned sequentially to the five segments. Within a segment, the order of the objects was randomized. For the firstdifferent condition, either the left-right orientation (Experiment 1) or the size (Experiment 2) was different in the first segment. For the fifthdifferent condition, the attribute was different in the fifth segment. Otherwise, objects were seen in the same orientation or size across segments. In the all-same condition, the attribute was the same in all segments. To ensure that memory for a subset of the changed objects in the firstdifferent and fifth-different conditions could be compared without confounding by overall study list position, the fifth segment of Block 2 was intermixed with the first segment of Block 3 , and the fifth segment of Block 3 was intermixed with the first segment of Block 4 .

Filler stimuli were also presented so as to maintain uniform spacing between repetitions and a constant mix of new and repeated objects throughout the list. For the beginning and ending buffer blocks, the spacing of repetitions ranged from 13 to 30 intervening items. For the three interior blocks, the spacing ranged from 17 to 30 items.

Three study lists were created in which the 45 objects used in Blocks 2-4 were rotated through each of the three conditions. For the size changes in Experiment 2, three additional lists were generated to counterbalance the direction of the size change (small-to-large and large-tosmall). Thus, there were three counterbalancing lists in Experiment 1, and six in Experiment 2 (which had equal numbers of large and small objects in all conditions).

The stimuli were presented on a white wall about $3 \mathrm{~m}$ in front of the subjects via an overhead projection panel driven by a Macintosh powerbook computer. All stimuli in Experiment 1, and the small stimuli in Experiment 2, occupied $\sim 4$ to $29 \mathrm{~cm}^{2}$ on the computer screen. To create the large stimuli in Experiment 2, sizes were increased $100 \%$ in both the vertical and horizontal directions. The large pictures occupied $\sim 17$ to $116 \mathrm{~cm}^{2}$ on the computer screen.
In what follows, we cail the single mirror-reversed or size-changed version of an object in the first-different or fifth-different conditions the "minority," and call the version that occurred four times in these conditions or five times in the all-same condition the "majority."

\section{Procedure}

Subjects were informed that they would see a series of objects and that their memory for these objects would later be tested, but the specific nature of the test was not described. Subjects were told that some of the objects would be repeated and that they should carefully attend to each presentation, even if it was a repetition. The 420 presentations were shown for $2 \mathrm{sec}$ each, with a .5 -sec interstimulus interval (ISI).

Following the study phase, each subject was given a test form containing 60 lines consisting of the trial number $(1-60)$ and three boxes, marked "left," "right," and "both." Subjects were informed that they would see two versions of some of the objects they had studied, on the left and the right side of the wall, differing in either orientation (Experiment 1) or size (Experiment 2). They were also told that they had seen some of the objects in only one version on all of its presentations, but they had seen other objects in both versions. Subjects were instructed that if they thought they had seen only the version of the object shown on the left or on the right, they should mark "left" or "right" accordingly; and that if they thought they had seen both versions of the object, they should mark "both." They were told to mark one and only one of the three choices on each line, even if they felt they were only guessing, and that they would have $5 \mathrm{sec}$ for each judgment. Test pairs were then presented for $5 \mathrm{sec}$ each with a 1-sec ISI. The majority version of the object appeared equally often on the left and the right, as did the large and small versions in Experiment 2.

\section{RESULTS}

Subjects could respond in three ways. They could choose the minority-only; the majority-only; or both versions of each object. Because the three response types are not independent (their proportions sum to 1), each response was analyzed in a separate one-way repeated measures analysis of variance (ANOVA). ${ }^{1}$ Two such analyses were conducted: one that compared all three conditions in all blocks, and one that directly compared the fifth-different condition in Blocks 2 and 3 with the first-different condition in Blocks 3 and 4 . The latter analysis controls for serial position because the study list positions of the minority stimuli in the two conditions completely overlapped. Subjects who gave the minorityonly response more than the majority-only response in the all-same condition were excluded because their errors exceeded correct responses in this condition. One subject was thus excluded from Experiment 1, leaving $N=$ 26; and 2 subjects were excluded from Experiment 2, leaving $N=25$.

\section{All Trials}

Here, we report data from all blocks. Means and standard deviations of response proportions are shown in Table 1.

Minority-only response. The minority-only response offers the strongest test of our hypothesis. In the firstdifferent condition, it means that subjects are reporting seeing only the version shown on the first presentation. In the fifth-different condition, it means they are reporting seeing only the version shown on the last presentation. In the all-same condition, it means that subjects are 
Table 1 Proportion of Response Types for All Trials

\begin{tabular}{|c|c|c|c|c|c|c|}
\hline \multirow[b]{2}{*}{ Condition } & \multicolumn{2}{|c|}{ Minority-Only } & \multicolumn{2}{|c|}{ Majority-Only } & \multicolumn{2}{|c|}{ Both } \\
\hline & $M$ & $S D$ & $M$ & $S D$ & $M$ & $S D$ \\
\hline \multicolumn{7}{|c|}{ Experiment 1 : Orientation Change } \\
\hline All-same & .15 & .08 & .64 & .15 & .22 & .12 \\
\hline First-different & .20 & .14 & .32 & .12 & .48 & .18 \\
\hline Fifth-different & .14 & .09 & .41 & .15 & .46 & .18 \\
\hline \multicolumn{7}{|c|}{ Experiment 2: Size Change } \\
\hline All-same & .06 & .09 & .59 & .24 & .35 & .22 \\
\hline First-different & .11 & .16 & .29 & .21 & .60 & .25 \\
\hline Fifth-different & .08 & .13 & .32 & .21 & .60 & .25 \\
\hline
\end{tabular}

Note-Response proportions may not sum to 1 because of rounding error.

reporting seeing the version that was never presented during the study phase.

The results were similar in Experiments 1 and 2. Subjects reported seeing only the single, minority presentation more often in the first-different condition than in the fifthdifferent condition in both experiments. Planned comparisons revealed significant differences $[F(1,50)=5.96$, $M S_{\mathrm{e}}=.008, p<.02$ for Experiment 1 and $F(1,48)=5.25$, $M S_{\mathrm{e}}=.003, p<.03$ for Experiment 2]. There was no reliable difference in the proportion of minority-only responses between the all-same condition and the fifth-different condition in either experiment ( $F<1$ in both cases).

Majority-only response. This response means that subjects are reporting seeing only presentations $2-5$ in the first-different condition and presentations $1-4$ in the fifthdifferent condition. In the all-same condition, majorityonly is the correct response.

Subjects gave the majority-only response in the fifthdifferent condition more often than the first-different condition. Planned contrasts revealed that this effect was reliable in Experiment $1\left[F(1,50)=5.56, M S_{\mathrm{e}}=.018, p<\right.$ $.02]$ but not in Experiment $2(F<1)$. As would be expected, subjects selected the majority-only response in the all-same condition significantly more often than in the first-different or fifth-different conditions $(p<.0001$ for both experiments).

Both response. Planned contrasts revealed that subjects correctly reported seeing both versions more often in the first-different and fifth-different conditions than in the all-same condition ( $p<.001$ in both experiments). The difference in this measure between the first-different and fifth-different conditions was not reliable in either experiment $(F<1)$.

\section{Serially Balanced Trials}

To rule out list position as a confounding factor, we next compared first-different and fifth-different conditions using only those trials in which study list positions of the changed versions were equated (see Method). This comparison excluded a third of the trials in each condition and therefore lowered the statistical power of the tests; hence, here we report $F$ values where $p<.1$. The means and standard deviations of these response proportions are given in Table 2.
Just as in the overall data, subjects reported seeing only the single, minority orientation when it occurred as the first presentation (first-different condition) more often than when it appeared as the final presentation (fifthdifferent condition). This difference was significant in Experiment $1\left[F(1,25)=6.81, M S_{\mathrm{e}}=.007, p<.02\right]$ and in Experiment $2\left[F(1,24)=7.03, M S_{\mathrm{e}}=.009, p<.01\right]$. This result confirms that the minority presentation is selected more often if it is the first than if it is the fifth presentation even when the overall position in the study list is controlled.

Subjects reported seeing only the majority orientation more often in the fifth-different condition than in the first-different condition. This effect was marginal for the orientation change $\left[F(1,25)=3.14, M S_{\mathrm{e}}=.025, p<.09\right]$ and not reliable for the size change $(F<1)$.

There were no reliable differences in the proportion of correct both responses between the two conditions in either experiment $(F<1)$.

\section{DISCUSSION}

These results confirmed that the attributes (orientation and size) of an early presentation of a repeated stimulus are learned better than are the attributes of later presentations. A primacy effect in memory for presentation 1 over presentation 5 was obtained in both experiments. We attribute the modest size of the effect to the small difference in familiarity (first vs. fifth presentation; because of the experimental design, more repetitions were impractical) and to our instructions to the subjects to attend to each presentation. It is, nevertheless, statistically significant.

These data might be explained in several ways, which vary in the degree and type of influence attributed to an object's first presentation. The most extreme interpretation is that the first presentation establishes a representation that is activated by subsequent presentations and tends to override perceptual information that would otherwise indicate that an attribute has changed. According to this view, there is a tendency to perceptually impose the attributes of the initial versions of the objects on later presentations. There is some experimental evidence that perceptual experience can be influenced by memory in this way. Jacoby, Allan, Collins, and Larwill (1988) showed that a single, prior presentation of a sentence can affect the perceived loudness of background noise in which the sentence is later embedded. Moreover, Goldstone (1995) has shown that the perceived color of a letter or digit is biased toward the known color range of other members of the same category. In both of these cases, recent exposures influenced an immediate perceptual judgment. We do not, of course, have direct evidence of such altered perception in our experiments.

A less extreme interpretation of these data is that the changed attribute is seen correctly, but that subsequent encoding into memory is distorted in the direction of the previous occurrences. Ausubel (1963) proposed a subsumptive process in which prior knowledge both en-

Table 2

Proportion of Response Types for Balanced List Position Trials

\begin{tabular}{|c|c|c|c|c|c|c|}
\hline \multirow[b]{2}{*}{ Condition } & \multicolumn{2}{|c|}{ Minority-Only } & \multicolumn{2}{|c|}{ Majority-Only } & \multicolumn{2}{|c|}{ Both } \\
\hline & $M$ & $S D$ & $M$ & $S D$ & $M$ & $S D$ \\
\hline \multicolumn{7}{|c|}{ Experiment 1: Orientation Change } \\
\hline First-different & .18 & .11 & .34 & .15 & .48 & .18 \\
\hline Fifth-different & .12 & .11 & .42 & .21 & .47 & .21 \\
\hline \multicolumn{7}{|c|}{ Experiment 2: Size Change } \\
\hline First-different & .14 & .14 & .29 & .17 & .57 & .21 \\
\hline Fifth-different & .07 & .09 & .33 & .18 & .60 & .23 \\
\hline
\end{tabular}

Note-Response proportions may not sum to 1 because of rounding error. 
hances the learning of a stimulus by anchoring it to a category and (paradoxically) obliterates the trace of the stimulus, leaving only the category type. A similar process has been suggested as an explanation of repetition blindness effects (Kanwisher \& Driver, 1992). These ideas are reminiscent of Piaget's assimilation mechanism, in which inputs are synthesized within existing cognitive structures (Piaget, 1952). Grossberg's (1987) adaptive resonance theory (ART) can be seen as a computational model that displays properties comparable to Piaget's assimilation. In ART, prior learning strongly influences the final state that the network will settle into, given a particular input.

The third and least extreme interpretation of our results is simply that less attention is directed to a familiar object than to a novel one. This classical view arises from Sokolov (1963), who proposed that a familiar stimulus elicits less attentional orienting and less physiological response than does a novel stimulus. In recent work, physiological attenuation of response to repetitions has been found in event-related potentials (Rugg, Soardi, \& Doyle, 1995), as well as in single-cell recordings in the "what" pathway of the monkey inferior temporal cortex (Baylis \& Rolls, 1987; Desimone, Miller \& Chelazzi, 1994). On the basis of neuroimaging studies, it has been suggested that the efficacy of memory encoding depends on novelty, as determined by areas of the brain that constitute a novelty detection network (Tulving, Markowitsch, Kapur, Habib, \& Sylvain, 1994).

There is cognitive as well as physiological evidence of attenuation of processing due to familiarity. The amount of time that subjects in a memory experiment devote to the study of a word (Rao \& Proctor, 1984) and the number of eye fixations they make to a picture (Hintzman, Summers, Eki, \& Moore, 1975) decrease with increasing familiarity. In both of these studies, familiarity appears to have attenuated the amount of attention paid to a stimulus. Familiar items may be filtered from further processing at an early stage of encoding that impairs later recognition memory (Tulving \& Kroll, 1995). As the data of Hintzman and Curran (1995; Hintzman et al., 1992) argue, however, repetitions are usually at least noticed, because frequency judgments increase monotonically even past the point where the learning of an attribute such as orientation has effectively stalled

A computational model that may help clarify these findings is outlined in Johnston and Hawley's (1994) "mismatch theory." In their model, if a stimulus is sufficiently similar to activate a stored representation, incoming attribute information can be actively inhibited. Presumably, the attributes of the stored representation are processed instead of the data-driven information. Johnston and Hawley have argued that the encoding of an item is strongly influenced by top-down conceptual information. In keeping with their analysis, our data suggest that even the first presentation of an item produces sufficient top-down influence on later trials to hinder the acquisition of additional data-driven information. The results of these two experiments hint at such top-down biases, but further research will be needed to determine the nature and strength of the mechanisms that are involved.

\section{REFERENCES}

AusubeL, D. P. (1963). The psychology of meaningful verbal learning An introduction to school learning. New York: Grune \& Stratton.

BAYLIS, G. C., \& Rolls, E. T. (1987). Responses of neurons in the inferior temporal cortex in short term and serial recognition memory tasks. Experimental Brain Research, 65, 614-622.

Culler, E., \& GiRdeN, E. (1951). The learning curve in relation to other psychometric functions. American Journal of Psychology, 64, 327-349.
Desimone, R., Miller, E. K., \& Chelazzi, L. (1994). The interaction of neural systems for attention and memory. In C. Koch \& J. L. Davis (Eds.), Large-scale neuronal theories of the brain (pp. 75-91). Cambridge, MA: MIT Press.

Estes, W. K. (1950). Toward a statistical theory of learning. Psychological Review, 57, 94-107.

Goldstone, R. L. (1995). Effects of categorization on color perception. Psychological Science, 6, 298-304

GrossberG, S. (1987). Competitive learning: From interactive activations to adaptive resonance. Cognitive Science, 11, 23-63.

Hintzman, D. L., \& CurRan, T. (1995). When encoding fails: Instructions, feedback, and registration without learning. Memory \& Cognition, 23, 213-226.

Hintzman, D. L., Curran, T., \& Oppy, B. (1992). Effects of similarity and repetition on memory: Registration without learning? Journal of Experimental Psychology: Learning, Memory, \& Cognition, 18, 667-680.

Hintzman, D. L., Summers, J. J., Ekı, N. T., \& Moore, M. D. (1975). Voluntary attention and the spacing effect. Memory \& Cognition, 3 , 576-580.

Jacoby, L. L., Allan, L. G., Collins, J. C., \& Larwill, L. K. (1988). Memory influences subjective experience: Noise judgments. Journal of Experimental Psychology: Learning, Memory, \& Cognition, 14, 240-247

Johnston, W. A., \& Hawley, K. J. (1994). Perceptual inhibition of expected inputs: The key that opens closed minds. Psychonomic Bulletin \& Review, 1, 56-72.

KANWISHER, N., \& DRIVER, J. (1992). Objects, attributes, and visual attention: Which, what, and where. Current Directions in Psychological Science, 1, 26-31.

PIAGET, J. (1952). The origins of intelligence in children. New York: International Universities Press.

RAO, K. V., \& Proctor, R. W. (1984). Study-phase processing and the word frequency effect in recognition memory. Journal of Experimental Psychology: Learning, Memory, \& Cognition, 10, 386-394.

RugG, M. D., Soardi, M., \& Doyle, M. C. (1995). Modulation of event-related potentials by the repetition of drawings of novel objects. Cognitive Brain Research, 3, 17-24.

SNodgrass, J. G., \& VANDERWART, M. A. (1980). A standard set of 260 pictures: Norms for name agreement, image agreement, familiarity, and visual complexity. Journal of Experimental Psychology: Learning, Memory, \& Cognition, 6, 174-215.

Sokolov, E. N. (1963). Perception and the conditioned reflex. New York: Macmillan

TUlving, E., \& KROLL, N. (1995). Novelty assessment in the brain and long-term memory encoding. Psychonomic Bulletin \& Review, 2 , 387-390.

Tulving, E., Markowitsch, H. J., Kapur, S., Habib, R., \& SylVAIN, H. (1994). Novelty encoding networks in the human brain: Positron emission tomography data. Neuroreport, 5, 2525-2528.

\section{NOTE}

1. The same analysis was done using an arc-sine transformation. These results led to the same conclusions, so only the analyses of proportions are presented.

(Manuscript received June 13, 1996; revision accepted for publication October $1,1996$. 